\title{
Deep space optical communications (DSOC) downlink simulation with varying PPM order
}

\author{
Emmanuel Domfeh Aboagye $^{1}$ (D) . Shun-Ping Chen ${ }^{1}$
}

Received: 29 January 2021 / Accepted: 3 September 2021 / Published online: 21 September 2021

(c) The Author(s) 2021

\begin{abstract}
During the course of a typical deep space mission like the Mars Earth mission, there exist a wide range of operating points, due to the different changes in geometry that consequently cause different link budgets in terms of received signal and noise power. These changes include distance range, Sun-Earth-Probe angle, zenith angle and atmospheric conditions. The different operating points, with different losses (background noise, pointing losses and atmospheric losses), lead to different capacities and data rates over the course of a typical deep space mission. Consequently, different engineering parameters are adjusted and optimized to combat some of these varying losses in order to get acceptable data rates and bit error probabilities. This is a useful reason to analyze and simulate various operating conditions that occur with the varying spatial orbital time periods of the resulting received signal power level, noise power level, capacity, data rates and bit error probabilities. This paper details results of simulations of typical deep space optical communication link operation.
\end{abstract}

Keywords Deep space optical communications (DSOC) $\cdot$ Pulse position modulation (PPM) · Photon counting · Intensity modulation · Photon efficiency · Channel capacity · Sun-Earth-Probe angle

Abbreviations
$\begin{array}{ll}\text { PPM } & \text { Pulse position modulation } \\ \text { APD } & \text { Avalanche photodiode } \\ \text { M-nary } & \text { PPM order } \\ \text { AU } & \text { Astronomical units } \\ \text { BEP } & \text { Bit error probability } \\ \text { SEP } & \text { Symbol error probability } \\ \text { BER } & \text { Bit error rate }\end{array}$

We declare that this manuscript presented is our own work. Areas or formulas used in the simulation that were quoted from other sources have their references provided.

Emmanuel Domfeh Aboagye

emmanuel.d.aboagye@stud.h-da.de; eadomfeh@yahoo.com

Shun-Ping Chen

shun-ping.chen@h-da.de

1 Institute of Communication Technologies, Darmstadt University of Applied Sciences, Birkenweg 8, D-64295 Darmstadt, Germany 
Tx Transmitter

Rx Receiver

DSOC Deep space optical communications

SEP angle Sun-Earth-Probe angle

M-PPM M-nary pulse position modulation

FOV Field of view

LLCD Lunar laser communications demonstration

\section{Introduction}

There has been a paradigm shift in the use of radio communications to optical communications in the field of deep space communications during the last decades. The reason for this increasing interest for the applications of optical communications in deep space is due to its unique attributes such as large bandwidth, license free spectrum, high data rate, efficient power utilization and low mass requirements.

Deep space optical communications (DSOC) involves a communication link that focuses mainly on the transmission between the distant planetary objects that are millions of kilometers apart from the Earth. One famous example is the communication link between the Earth and a spacecraft surrounding another planet such as Mars. The distance range between these planetary objects may be described in astronomical units (AU).

This paper details results of simulations conducted in a typical DSOC Mars-Earth link operation. During the course of a typical Mars-Earth mission, there exist a wide range of operating points due to the different changes in geometry that consequently leads to different Mars-Earth link budgets in terms of received signal and noise power. Examples of these parameters during the course of a Mars-Earth mission include distance range, Sun-EarthProbe angle (SEP angle), zenith angle and atmospheric conditions. These different operating points can cause different losses (background noise, pointing losses and atmospheric losses) leading to different channel capacities and data rates over the course of a typical Mars-Earth mission. Consequently, different parameters are adjusted to combat some of these varying losses in order to get acceptable data rates and bit error probabilities. Thus, over the course of a typical Mars-Earth mission, there will be varying noise power levels and received signal power levels due to the varying orbital time periods. For example, there will be orbital time instances when the Sun will be between Mars and Earth leading to higher level of noise due to the small SEP angle and also higher distance range between the Mars and the Earth (the largest distance between Earth and Mars is about 225 million km) (Biswas et al. 2004). Conversely, in other orbital time instances, there will be situations where the Sun will not be between mars and earth. This causes lower level of noise and also lower distance range between Mars and Earth (thus the closest distance of Earth to Mars is 56 million km) (Biswas et al. 2004). The ratio of the ranges of these two distinct positions over an orbital period (2.2 years) is approximately 4 . All these factors lead to different channel capacities, data rates and bit error probabilities in these different conditions. Different engineering input parameters are adjusted accordingly to maintain or improve the data rates and bit error rates as the spatial orbital time period varies.

This provides a good basis to undertake analyses and simulations of the various operating conditions that occur with the varying spatial orbital time periods on the resulting received signal power level, noise power level, capacity, data rates and bit error probabilities. 
In the subsequent pages of this paper, a deep space optical communication system is modeled and simulated by using Matlab. The impact of optimization of the number of received signal photons and noise photons, channel capacity, data rates and bit error rates through tuning and adjustment of the various parameters such as PPM order, laser transmitter aperture size, receiver aperture size and laser transmit power is also analyzed in this paper. A photon counting model in a non-coherent detection system setup for intensity modulation was used in these simulations to determine the photon counts at the receiver side and consequently recover the data transmitted. This is because non-coherent detection is the best for energy efficiency which is indispensable in space. Furthermore, PulsePosition Modulation (PPM) was used in the downlink simulations due to higher energy efficiency with low duty cycles compared with On-Off-Keying (OOK) in deep space when transmitting from Mars to Earth. (Helstrom 1976).

\section{DSOC systems}

\subsection{DSOC model block diagram}

The deep space optical communication DSOC system model consists of the laser transmitter (Tx), Tx aperture gain, DSOC channel, receiver $(\mathrm{Rx})$ aperture gain and the APD receiver $(\mathrm{Rx})$. Figure 1 shows a block diagram of the model. The DSOC channel comprises space loss, pointing losses, atmospheric effects and optical background of the link between the transmitters and receivers stationed on Earth and Mars (Hemmati 2006).

\subsection{DSOC model equations}

In M-PPM, each symbol interval is divided into $\mathrm{M}$ time slots and a non-zero optical pulse is placed in one of these time slots while other slots are kept vacant. Moreover, for the purpose of synchronization additional slots called synchronization slots are added. For long distance or deep space communications, M-PPM scheme is widely used because it provides a high peak-to-average power ratio (PAPR) that improves its average-power efficiency (Chen 1992). Since k bits are sent per symbol, the average number of signal counts required per bit is divided by $\mathrm{k}$, which makes it more energy efficient than OOK modulation for $\mathrm{k}>2$. Furthermore, unlike OOK, M-PPM does not require an adaptive threshold. However, the M-PPM scheme has poor bandwidth efficiency at higher values of M. Downlink simulations were done for PPM orders ranging from 2 to 4096; nevertheless 4 to 256 is typically used in the deep space mission for Mars (Hemmati 2006).

The average power for M-PPM can be calculated as:

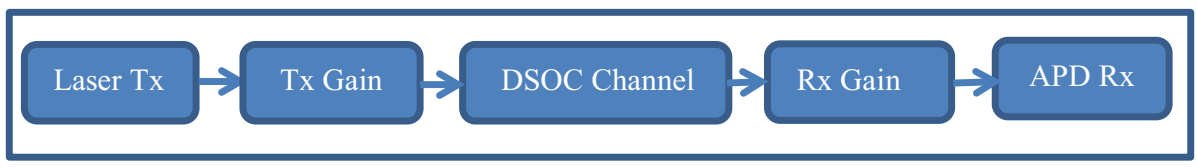

Fig. 1 Block diagram of a DSOC model 


$$
P_{\mathrm{avg}}=\frac{\left(P_{\max }+P_{\min } \cdot(\mathrm{M}-1)\right)}{\mathrm{M}}
$$

The signal slot width $T s$ is given by the equation depending on the parameter $B R$ :

$$
T s=\frac{1}{P R R}
$$

The pulse repetition rate $P R R$ required for a system with PPM order $\mathrm{M}$, bit rate $B R$ and $\mathrm{k}$ bits per symbol is:

$$
P R R=\frac{M \cdot B R}{k}
$$

The free space loss FSL is dependent on the distance (range) d between the transmitter and receiver. FSL is:

$$
F S L=20 \log \left(\frac{4 \pi d}{\lambda}\right)
$$

The transmitter aperture gain (Tx gain) and receiver aperture gain (Rx gain) are dependent on the telescope aperture diameter $D$ in meters, the optical wavelength $\lambda$ in meters and the optical efficiency $\eta$ of the lens. The gains are (Arnon et al. 1997) in the equation:

$$
G=\eta\left(\frac{\pi D}{\lambda}\right)^{2}
$$

The background radiation interferes with the received signal. Objects producing light, such as the Sun, the stars and Earth, may interfere with the received signal and cause background noise. Received background power $P_{b a c k}$ depends on the background irradiance, effective receiver area, receiver field of view, optical filter bandwidth and the optical efficiency and it is modeled as:

$$
P_{\text {back }}=H_{b} \cdot A_{\text {rec }} \cdot \eta \cdot \gamma_{F O V} \cdot \Delta \lambda_{n r}
$$

where $H_{b}$ is the background irradiation energy density $\left(\mathrm{W} / \mathrm{m}^{2} / \mathrm{sr} / \mu \mathrm{m}\right), A_{\text {rec }}$ is the effective receive area $\left(\mathrm{m}^{2}\right), \gamma_{F O V}$ is the receiver field of view measured in steradians (sr), $\Delta \lambda_{n r}$ is the optical filter bandwidth $(\mu \mathrm{m})$ and $\eta$ is the optical efficiency of the receiver (scalar). The receiver field of view is:

$$
\gamma_{F O V}=\frac{\pi}{4} \theta_{F O V}^{2}
$$

where $\theta_{\mathrm{FOV}}$ represents the planar angular detector field of view (radians) and depends on the telescope aperture. The value of sky radiance, $H_{b}$ ranges from 0.007 to $0.015 \mathrm{~W} /\left(\mathrm{cm}^{2} \mathrm{sr}\right.$ $\mu \mathrm{m})$ depending on the zenith angle and the time of daylight (Hemmati 2006).

Adverse atmospheric effects impact on the performance of the optical link. Consequently, during the design of the ground station receiver system, measures are factored into the process in order to reduce the effects of adverse atmospheric conditions. These atmospheric effects include: atmospheric scintillation, atmospheric irradiance fluctuations and atmospheric turbulence induced angle of arrival jitter fluctuations, atmospheric transmittance and atmospheric "seeing". One method is the use of large effective receiver aperture diameter during the design of the DSOC downlink system in order to collect more photons. The side-effect associated with such design is the increased impact of background noise 
through sky radiance. This is factored into the background noise calculation through the value specified for sky radiance. The negative side-effect of using large receiver aperture diameter is also minimized by restricting the detector field of view (FOV) in order to reject excessive background noise from sky radiance. Moreover, special spectral filters are used to reduce the collected sky radiance or background flux. Another technique for reducing the sky radiance is the use of a form of distributed optical antenna array at the receiver of the ground station.

Furthermore, the negative impact of adverse atmospheric turbulence is reduced by employing large aperture averaging for the receiver with diameter ranging 5-10 $\mathrm{m}$ whilst Fried parameter $r_{0}$ is $0.05 \mathrm{~m}$. This provides high $D_{r x} / r_{0}$ that reduces the impacts of atmospheric scintillation, irradiance fluctuations, "seeing effects" and induced angle of arrival jitter fluctuations according to pages 184-207 of literature (Hemmati 2006). Atmospheric scintillation penalty $(0.1 \mathrm{~dB})$ is assumed in the link budget.

Atmospheric transmission loss is:

$$
T_{\text {atm }}=e^{M_{r} \cdot \ln \left(T_{\text {zenith }}\right)}
$$

where $T_{\text {zenith }}$ is atmospheric zenith transmittance and $M_{r}$ is relative air mass.

Other factors like the choice of location of ground station and the time (day or night) for receiving data on the downlink of the DSOC system is very vital in mitigating against some of the adverse atmospheric impacts. Pages 169-184 of literature in Hemmati (2006) subtitled "3.3 Atmospheric Issues on Ground Telescope Site Selection for an Optical Deep Space Network" provide an overview of mitigating some of the impacts of the atmospheric turbulence effects. For example, the altitude of the location of ground station and zenith angle during the downlink designs. Thus, ground station locations are carefully chosen in order to limit the impact of atmospheric effects and losses as low as possible. Finally, the choice of $1.55 \mu \mathrm{m}$ as the optical wavelength for the downlink of the DSOC system help further mitigate against atmospheric losses such as absorption and scattering of the light beam due to gas molecules and aerosols present in the atmosphere.

Incident light at the APD is converted into electrical signals proportional to the power and the responsivity is:

$$
R=\frac{q \cdot n}{h \cdot f}
$$

where, $q$ is the electron charge, $n$ the quantum efficiency, $h$ the Planck's constant and $f$ is optical frequency.

A stream of discrete photons are emitted from a laser transmitter and collected by the APD receiver. In order to build a realistic APD receiver, noise originates within the diode was considered. Shot noise depends on the average current from different sources and is modeled by:

$$
\sigma_{s n}^{2}=2 \cdot q \cdot R \cdot \operatorname{Pr} \cdot G^{2} \cdot F \cdot B w
$$

where $G$ is the optical amplifier gain, $\operatorname{Pr}$ is the incident received optical power, $B w$ is the electrical bandwidth, $F$ is the excess noise factor of the APD and is:

$$
F=k_{e f f} \cdot G+\left(1-k_{e f f}\right)\left(2-\frac{1}{G}\right)
$$

where $k_{\text {eff }}$ is the ratio of the hole and electron ionization coefficients. 
Background shot noise produced by a similar process as signal shot noise is:

$$
\sigma_{b g}^{2}=2 \cdot q \cdot R \cdot P_{b a c k} \cdot G^{2} \cdot F \cdot B_{w}
$$

where $P_{\text {back }}$ represents the background noise power.

The dark current shot noise is:

$$
\sigma_{d c}^{2}=2 \cdot q \cdot F \cdot G^{2} \cdot I_{b} \cdot B_{w}+2 \cdot q \cdot I_{s} \cdot B_{w}
$$

where $I_{b}$ is the bulk leakage current which becomes amplified and $I_{s}$ is the surface leakage current which does not pass through the avalanche region.

The thermal noise is:

$$
\sigma_{t h}^{2}=\frac{4 \cdot k_{B} \cdot T_{r} \cdot F \cdot B_{w}}{R_{L}}
$$

where $R_{L}$ is the load resistance, $T_{r}$ the electronic system noise temperature, and.

$k_{B}$ the Boltzmann constant. The temperature $T_{r}$ is the equivalent temperature of the loss and is usually the physical temperature of the load resistor.

Aggregating all currents and noise current sources, the mean and variance of the total output from the receiver can be derived. Two scenarios are considered: firstly when a signal pulse is received and secondly when there is no pulse received between the pulses. The mean output current from the receiver for both cases is:

$$
\begin{gathered}
\mu_{1}=R \cdot G \cdot P_{r 1}+R \cdot G \cdot P_{b}+G \cdot I_{b}+I_{s} \\
\mu_{0}=R \cdot G \cdot P_{r 0}+R \cdot G \cdot P_{b}+G \cdot I_{b}+I_{s}
\end{gathered}
$$

where the index "1" denotes a received pulse and a " 0 " denotes the absence of a pulse. The variance of the output current is:

$$
\begin{aligned}
& \sigma_{1}^{2}=\sigma_{s n 1}^{2}+\sigma_{b g}^{2}+\sigma_{d c}^{2}+\sigma_{t h}^{2} \\
& \sigma_{0}^{2}=\sigma_{s n 0}^{2}+\sigma_{b g}^{2}+\sigma_{d c}^{2}+\sigma_{t h}^{2}
\end{aligned}
$$

The signal to noise ratio (SNR) is determined and the bit error rate is calculated from these variances and mean output currents (Gallager 1968; Hamkins 2004).

The capacity of the DSOC system was determined from equation given by Wyner (Wyner 1988) and Pierce (Pierce et al. 1981) in page 93 of Hemmati (2006) as:

$$
C=\left(\log _{2} e\right) \frac{\lambda}{M}\left[\left(1+\frac{1}{\rho}\right)(\operatorname{In}(1+\rho))-\left(1+\frac{M}{\rho}\right) \operatorname{In}\left(1+\frac{\rho}{M}\right)\right]
$$

$\rho=\frac{\lambda_{S}}{\lambda_{B}}$ is the (detected) peak received signal power to background power (Hemmati 2006).

The data rate, $\mathrm{R}$ is:

$$
R=\frac{C \cdot \log _{2} M}{M}
$$

where $\mathrm{C}$ is the channel capacity and $\mathrm{M}$ is the PPM-order.

Data throughput is: 


$$
\text { Throughput }=R \cdot \text { Code_Rate }
$$

Simulations of the received signal photons, noise photons, channel capacity, data rate and data throughput are also computed accordingly (Hemmati 2006; Hamkins 1999). All equations were obtained from reference (Hemmati 2006). Table 1 gives the list of parameters and values used for the simulation (Biswas and Piazzolla 2003). Synchronization slots were not considered in the simulations. Bit SNR is explained as slot SNR divided by the number of bits in a PPM Symbol derived in pages 258-259 of reference (Hemmati 2006).

\section{Simulation results and analysis}

Simulations were done to investigate the impact of a varying PPM order $(M=2,4,8,32$, $64,128,256,512,1024,2048$ and 4096) on the number of received signal photons, noise photons, channel capacity, bit error probability, bit error rate and data rates in relation to the distance range dependency. All other parameters remained unchanged for the simulation. The figures below show the results of the simulations.

Figure 2 below shows that the number of detected signal photons decrease with increasing distance for a particular constant PPM order. Concurrently, the figure also depicts that

Table 1 Parameters for simulations

\begin{tabular}{|c|c|c|c|}
\hline Name & Symbol & Value & Unit \\
\hline Planck's constant & $H$ & $6.624 \times 10^{-34}$ & Joules/Hertz \\
\hline Electron charge & $Q$ & $1.6 \times 10^{-19}$ & Coulombs \\
\hline Boltzmann const & $k B$ & $1.38 \times 10^{-23}$ & Joules/Kelvin \\
\hline Load resistance & $\mathrm{R}_{\mathrm{L}}$ & $5.75 \times 10^{12} \times \mathrm{Ts}$ & Ohm \\
\hline Elevation & $\theta_{F O V}$ & 20 & Degrees \\
\hline Signal slot width & $T_{\mathrm{s}}$ & Variable & Seconds \\
\hline Laser Tx power & $\mathrm{P}_{\mathrm{avg}}$ & 5.0 & Watt \\
\hline Tx aperture & $\mathrm{D}_{\mathrm{tx}}$ & 0.22 & Meters \\
\hline Rx aperture & $\mathrm{D}_{\mathrm{rx}}$ & 5.0 & Meters \\
\hline Link margin & $\mathrm{L}_{\text {margin }}$ & 3.0 & $\mathrm{~dB}$ \\
\hline Optical efficiency & $\mathrm{H}$ & 0.35 for Detector & \\
\hline Pointing loss & $\mathrm{L}_{\text {Point }}$ & 2 & $\mathrm{~dB}$ \\
\hline Transmission loss & $\mathrm{L}_{\text {Trans }}$ & 0.55 & \\
\hline Wavelength & $\lambda$ & 1.55 & $\mu \mathrm{m}$ \\
\hline PPM order & $M$ & Variable & \\
\hline Bits per symbol & $k$ & Variable & bits \\
\hline Sky radiance & $H_{b}$ & 0.0085 & $\mathrm{~W} /\left(\mathrm{cm}^{2} \mathrm{sr} \mu \mathrm{m}\right)$ \\
\hline $\begin{array}{l}\text { Atmospheric } \\
\text { zenith transmit- } \\
\text { tance }\end{array}$ & $T_{\text {zenith }}$ & 0.95 & \\
\hline Relative air mass & $M_{r}$ & 2.9 & \\
\hline Code rate & Code_Rate & $1 / 3$ & \\
\hline Fried parameter & $r_{0}$ & 0.05 & Meters \\
\hline Scintillation loss & $L_{\text {scint }}$ & 0.1 & $\mathrm{~dB}$ \\
\hline Bit rate & $B R$ & 100 & Mbit/s \\
\hline
\end{tabular}


Fig. 2 Photons/pulse versus distance

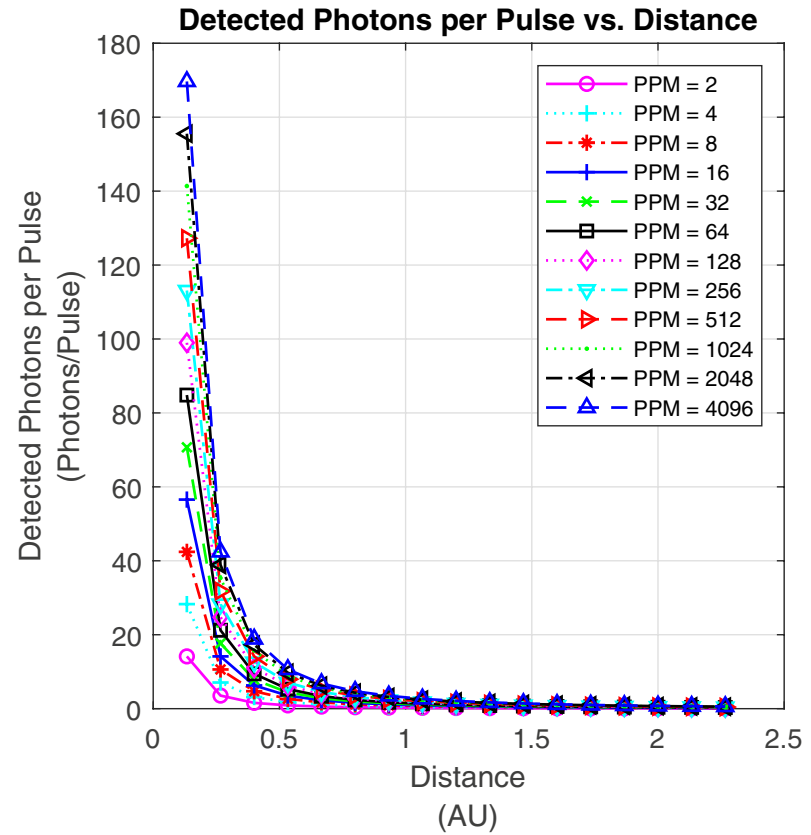

for a specific constant distance range, higher PPM orders give rise to higher photon counts. Figure 3 below gives a 3D pictorial view of the interrelationship between channel capacity and BEP with varying distance range for different values of PPM order.

Figures 4 and 5 as shown below indicate how the bit error rate (BER) varies with the photons/pulse and photons/slot respectively for different PPM orders. It can be observed that for a constant PPM order, BER decreases with increasing photons/pulse and photons/slot respectively. Moreover, as Hamid explained in page 254 of reference (Hemmati 2006), with the presence of background noise, the dependence on $M$ becomes evident as the BER performance for increasing $M$ degrades. Consequently, it is not appropriate to interpret BER performance with only photons/pulse as a measure of power efficiency as Fig. 4 shows. Thus, the use of the average photons/slot as a measure of power efficiency, as shown in Fig. 5, that takes into account the dependence of $M$ when BER is plotted against photons (measure of power efficiency) gives an extra insight into the BER performance. This explains the differences in the charts from Figs. 4 and 5 shown below. Whereas low values of PPM order (M) in Fig. 4 produces a lower BER compared to high values of PPM order (M), the contrasting scenario as shown in Fig. 5 occurs for different values of PPM order (M). The complete analysis, that takes into account the dependence on $\mathbf{M}$, when measuring BER performance versus average power efficiency in terms of photons as shown in Fig. 5, indicates that higher PPM orders (M) give better BER performance. Thus, taking a particular photons/slot (a measure of average power efficiency) in Fig. 5 into consideration, higher PPM orders (M) provide lower BER. Similar explanation is attributed to charts from Figs. 6 and 7 for BEP performances.

Figures 8 and 9 below respectively show the dependence of bit error rate (BER) and bit error probability (BEP) on distance for various values of PPM order (M). It can be observed that for a specific constant PPM order (M), BER and BEP increase with increasing distance. Moreover, it can be inferred that for a specific constant distance, higher values 


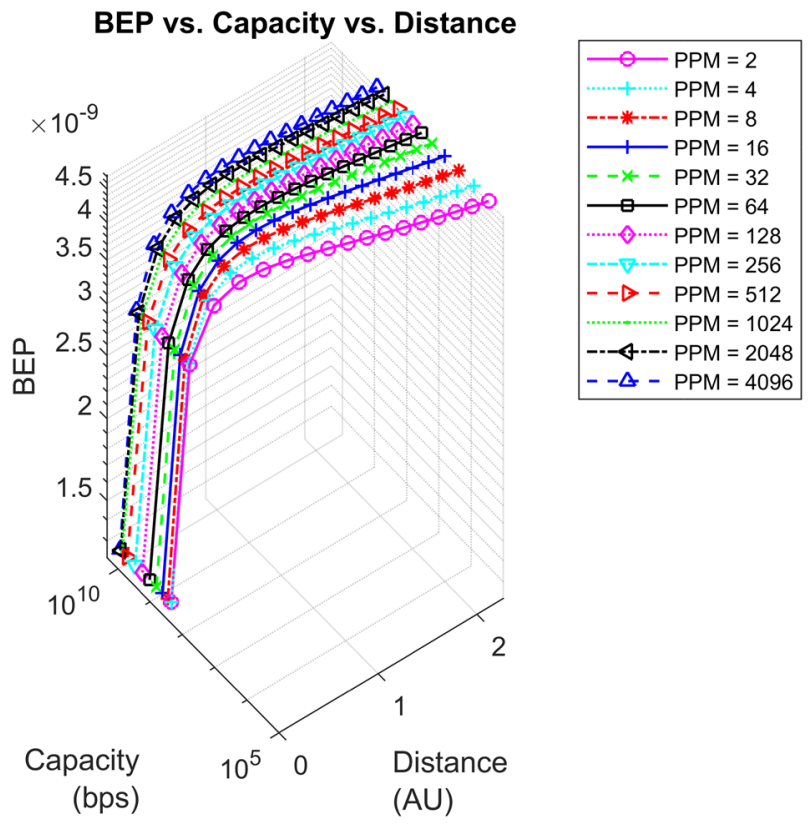

Fig. 3 BEP, capacity versus distance

Fig. 4 BER versus photons/pulse

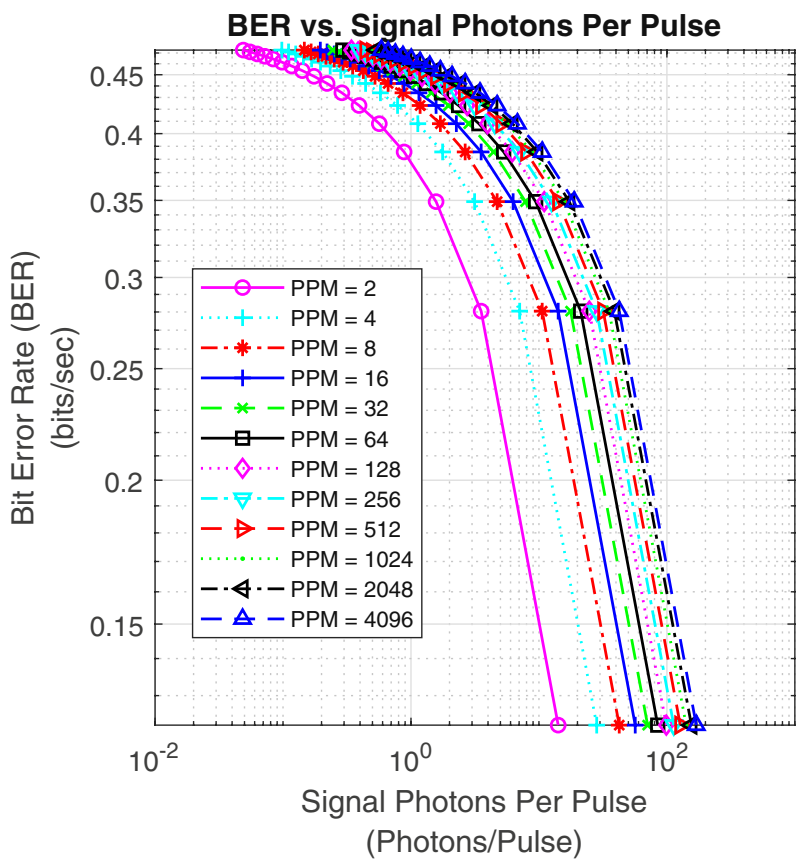


Fig. 5 BER versus photons/slot

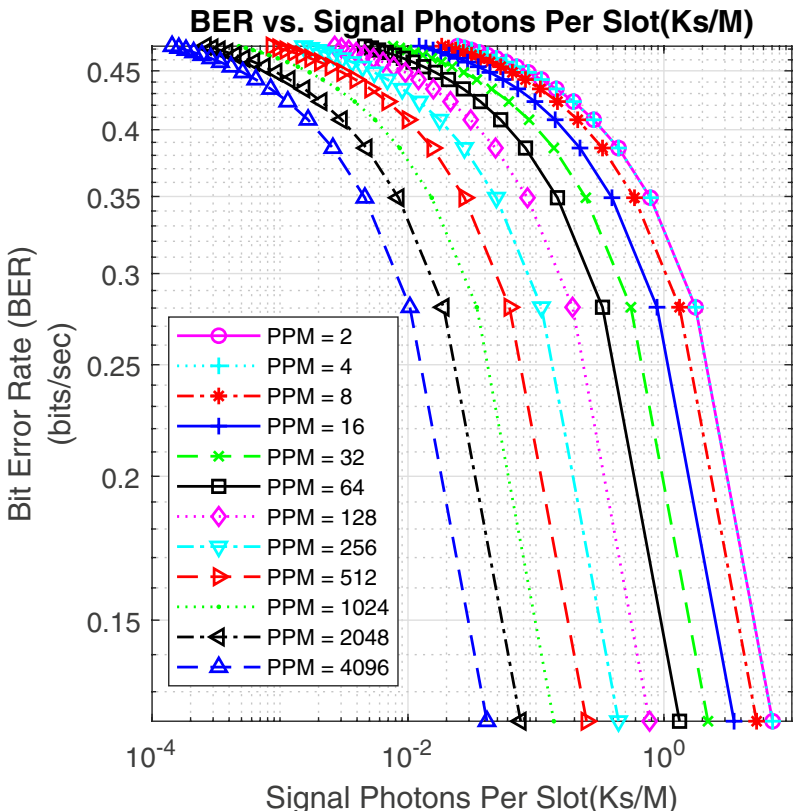

Signal Photons Per Slot(Ks/M)

(Photons per Slot)

Fig. $6 \mathrm{BEP}$ versus photons/pulse

BEP vs. No. of Signal Photons Per Pulse

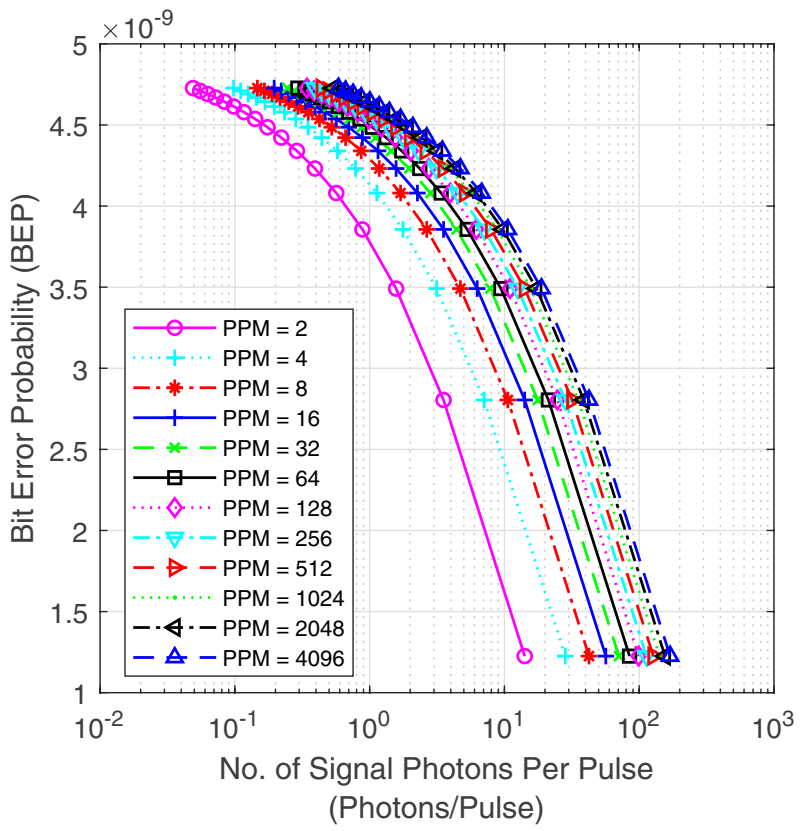


Fig. 7 BEP versus photons/slot

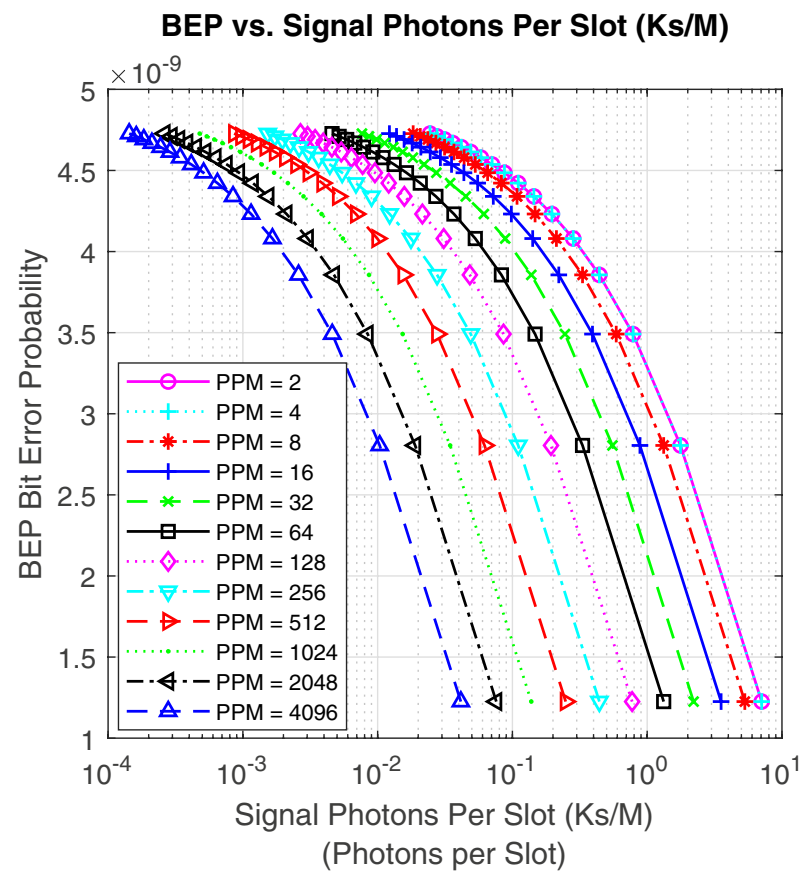

of PPM order (M) provide lower BER and BEP in simulations of varying PPM order (M) as shown in Figs. 8 and 9 respectively. These charts and analyses are further explained in Figs. 5 and 7 where higher PPM orders (M) provided lower BER and BEP respectively.

The signal to noise ratio (SNR) dependence on distance was determined by plotting slot SNR and bit SNR against the distance respectively in Figs. 10 and 11 for various PPM orders (M). Slot SNR is the signal to noise ratio for a symbol as a whole whereas bit SNR takes into account the number of bits in the symbol. According to Hemmati (2006), slot SNR is calculated with the formula $K_{s}^{2} / K_{b}$ whereas bit SNR is calculated using the formula $K_{s}^{2} /\left(2 K_{b} \cdot \log _{2} M\right)$ for any given set of values of signal photons $\left(K_{s}\right)$, background photons $\left(K_{b}\right)$ and PPM order (M). Consequently, slot SNR shows less dependence on PPM order (M). Furthermore, the charts in Figs. 10 and 11 show that for a specific constant PPM order (M), both slot SNR and bit SNR decrease with increasing distance. Moreover, it can be inferred from the results in Figs. 10 and 11 below that considering a specific constant distance, there exist an average of a $30 \mathrm{~dB}$ gap (Hemmati 2006) between SNR for simulations when varying PPM order from $M=2$ and $M=4096$. Thus, higher PPM orders have higher slot SNR and bit SNR for a specific constant distance.

The PPM symbol error probability (SEP) versus photon/slot is shown below in Fig. 12 whereas Fig. 13 exhibits the PPM symbol error probability (SEP) dependence on the distance. According to references (Hemmati 2006; Hamkins 2004; Hughes April 1992), the PPM symbol error probability (SEP) relates to bit error probability by the equation given in page 254 of Hemmati (2006) as $P_{b}=0.5 P_{s} \cdot M /(M-1)$. Figure 12 indicates that for a specific constant PPM order (M), SEP decreases with increasing photons/slot. Also, 
Fig. 8 BER versus distance

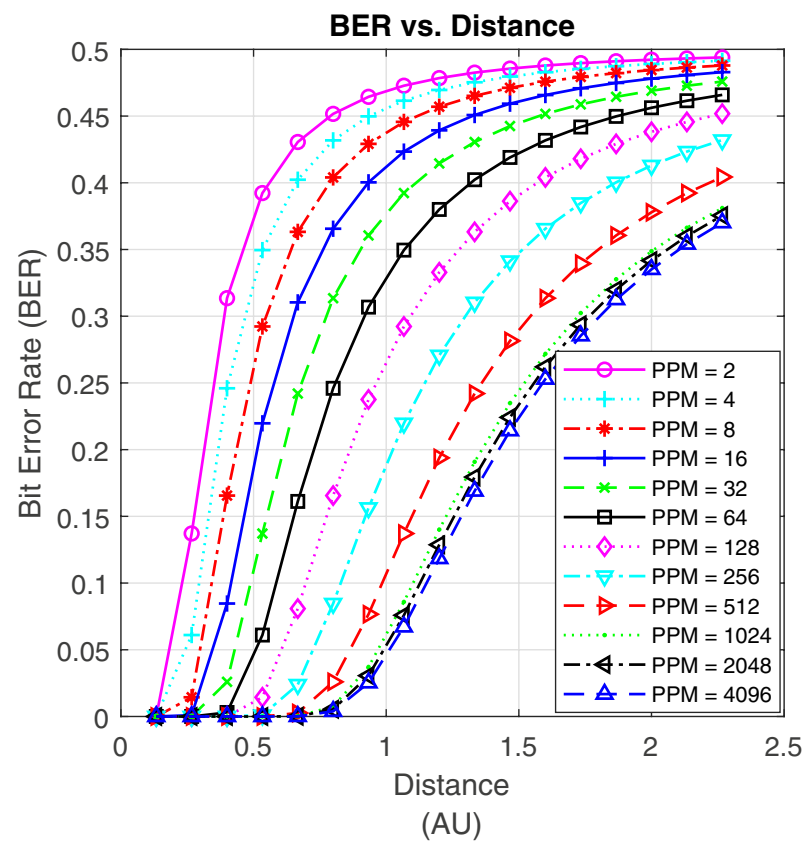

Fig. 9 BEP versus distance

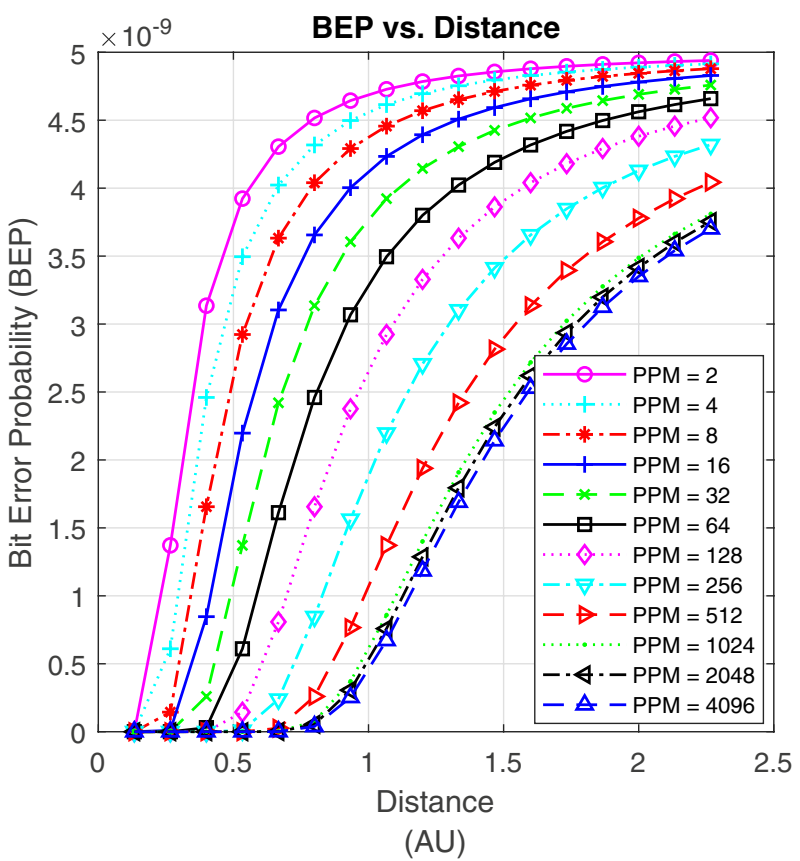

considering a specific constant photons/slot in Fig. 12, it is observed that the SEP decreases with increasing PPM order from $M=8$ to $M=4096$. Moreover, it can be inferred from Fig. 13 that SEP increases with increasing distance. Also, considering a specific constant 
Fig. 10 Slot SNR versus distance

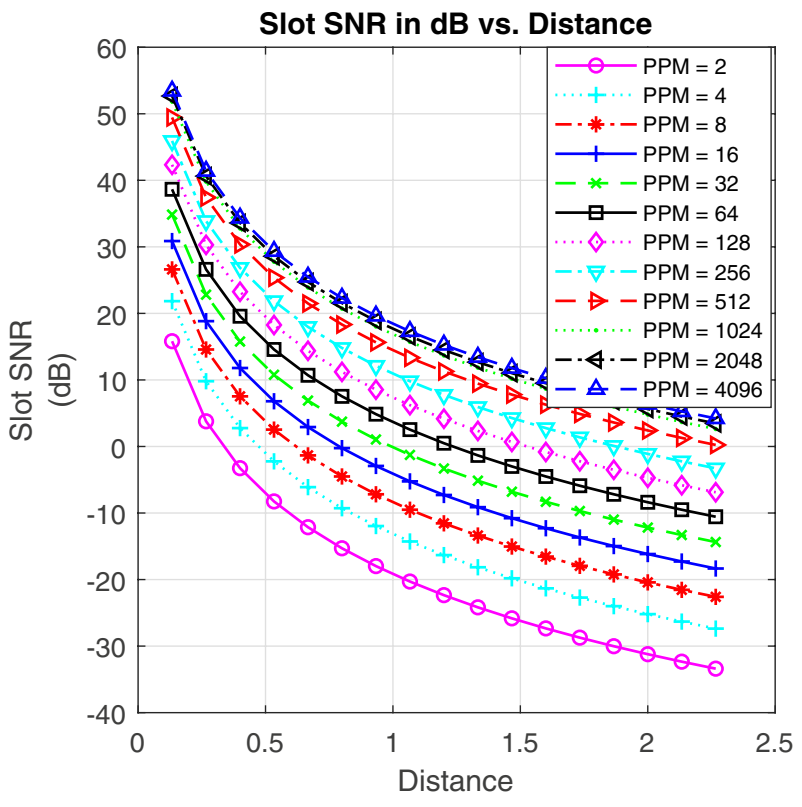

$(\mathrm{AU})$

Fig. 11 Bit SNR versus distance

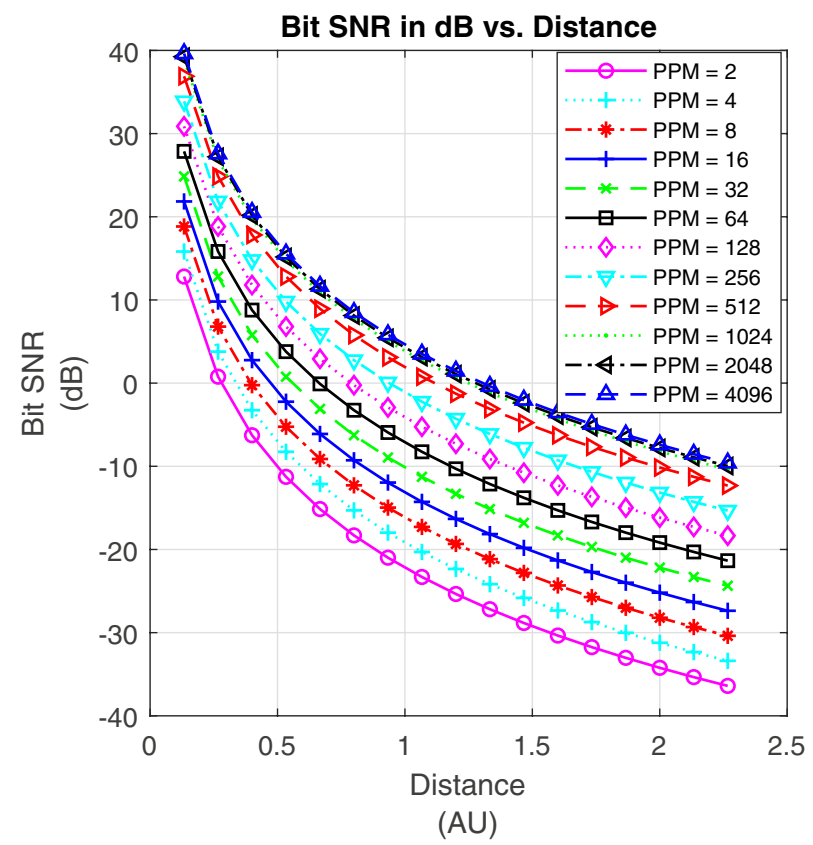

distance in Fig. 13, it can be seen that the SEP decreases with increasing PPM order from $\mathrm{M}=8$ to $\mathrm{M}=4096$. 
Fig. 12 SEP versus photons/slot

SEP vs. Signal Photons Per Slot (Ks/M)

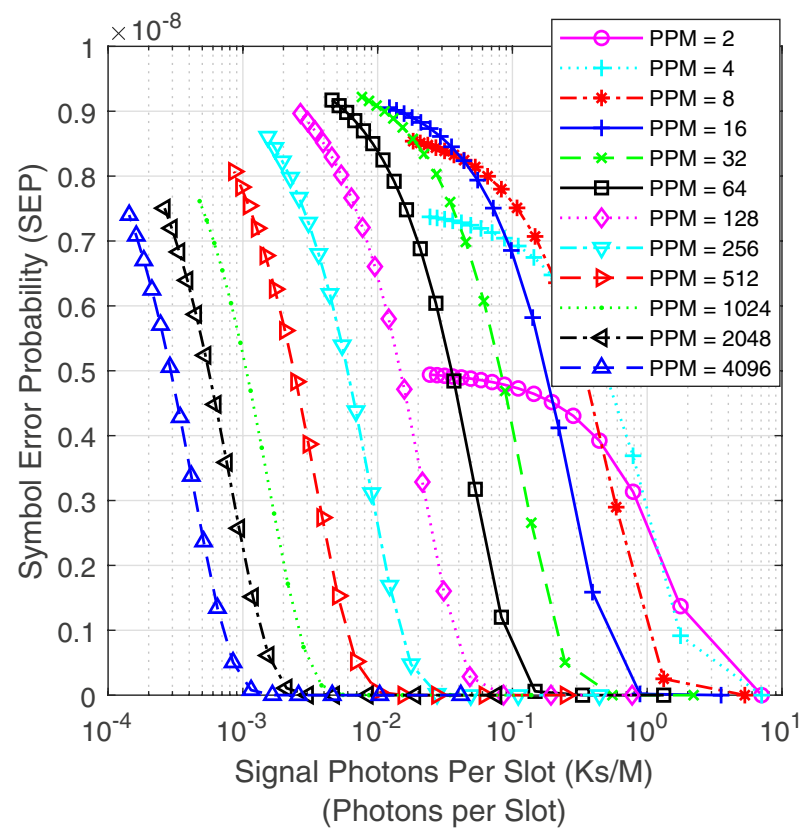

Fig. 13 SEP versus distance

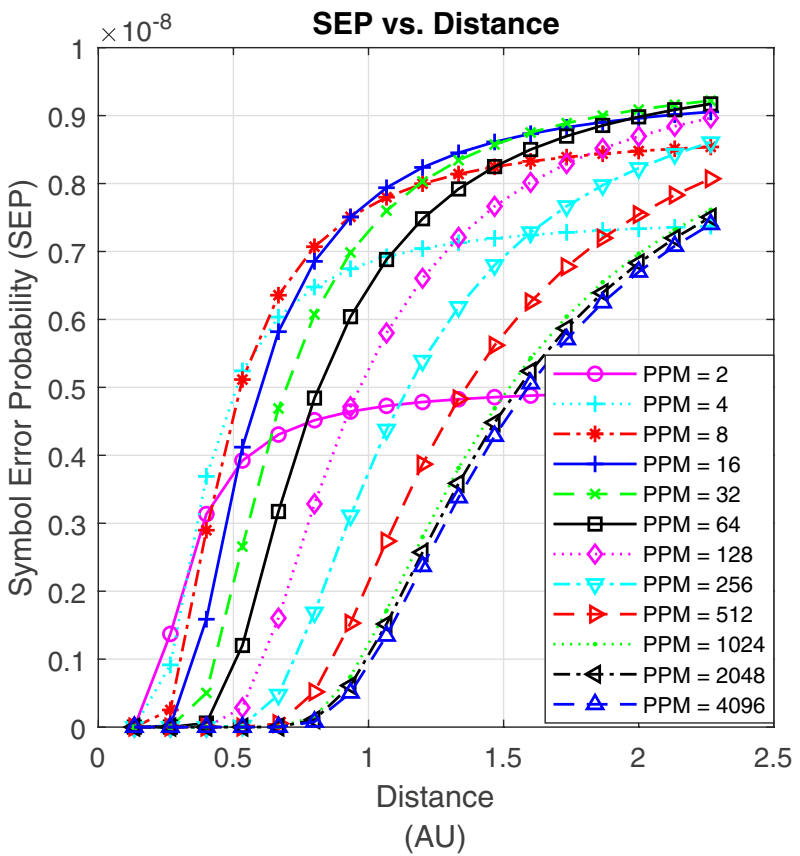

Figure 14 below shows that the transmit intensity (based on the peak power per symbol) varies according to the PPM order whereas Fig. 15 shows that the effective area of 
Fig. 14 Tx intensity versus distance
Tx Intensity based on peak power vs. Distance

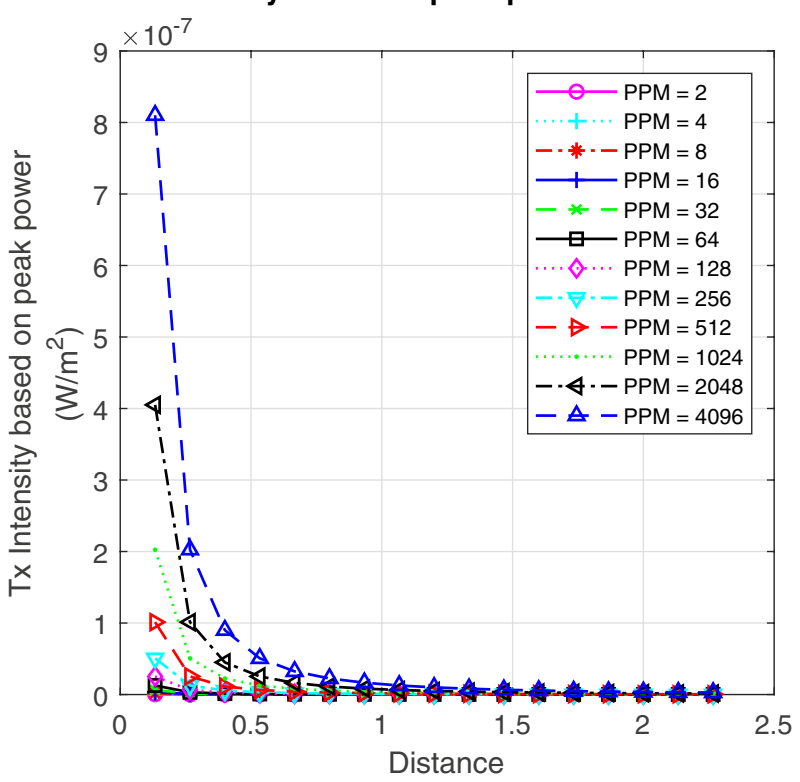

$(\mathrm{AU})$

divergence is unaffected by the variation of the PPM order. Concurrently, it can be seen that the transmit intensity of the laser reduces with increasing distance whereas the effective area of divergence increases with increasing distance.

The capacity of the optical channel is a very important reference value for determining the useful data rates achievable with any modulation and coding scheme (Hemmati 2006). Thus, the data rate can be determined directly from the channel capacity taking into consideration the code rate factor; hence the need to determine how the optical channel capacity changes with varying PPM order (M). According to Hemmati (2006), the capacity of the optical channel is a function of the received optical signal and noise powers, the modulation and the detection method. A hard-decision capacity is considered whereby the receiver makes estimates of each PPM symbol, passing these estimates, or hard decisions, on to the decoder. Consequently, the (hard-decision) capacity can be expressed as well as a function of the probability of symbol error Ps. Figure 16 shows that the optical channel capacity increases with increasing average received power. Considering the chart produced by each PPM order, Fig. 16 also exhibits three segments for the plot of channel capacity versus average received power namely: noise-limited capacity (in the form of quadratic plot in the beginning of the chart), quantum-limited capacity (in the form of linear plot in the middle of the chart) and the bandwidth-limited capacity (in the form of a saturated or constant plot at the tail-end of the chart) following in this order along an increasing trend of the average received power. According to Hemmati (2006); Moision and Hamkins 2003), there is a peak constraint in the power resulting in an upper limit on the PPM order and hence the upper limit (saturation) in optical channel capacity.

Figures 17, 18 and 19 respectively show how the optical channel capacity, data rate and data throughput change with distance for various values of PPM order (M). Generally, for a specific constant PPM order (M), the optical channel capacity, data rate and data throughput decrease with increasing distance as shown in Figs. 17, 18 and 19 below. Moreover, 
Fig. 15 Divergence versus distance

Fig. 16 Capacity versus received power
Effective Area of Divergence vs. Distance

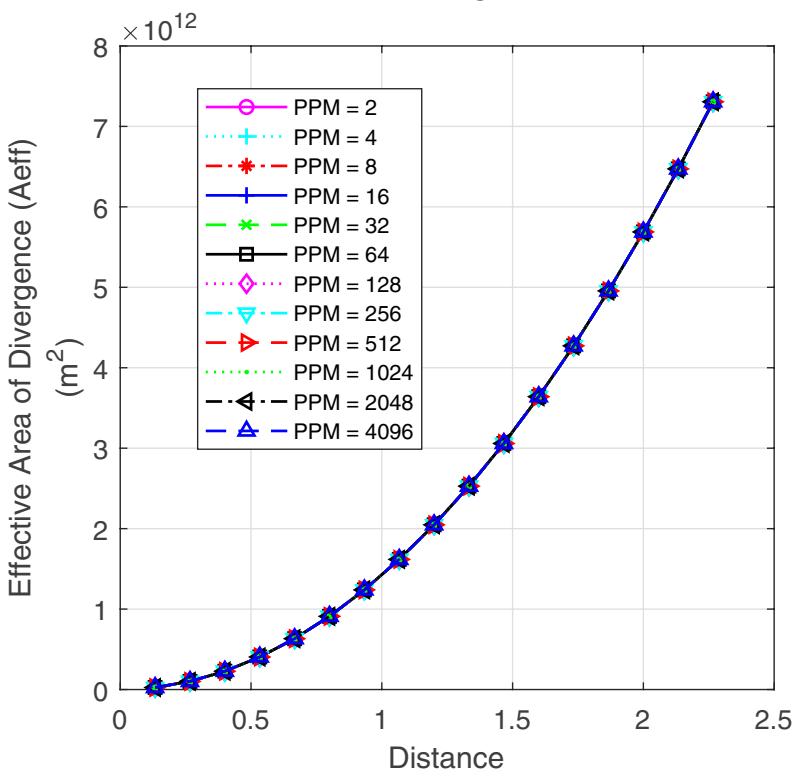

$(\mathrm{AU})$

Channel Capacity vs. Recieved Power

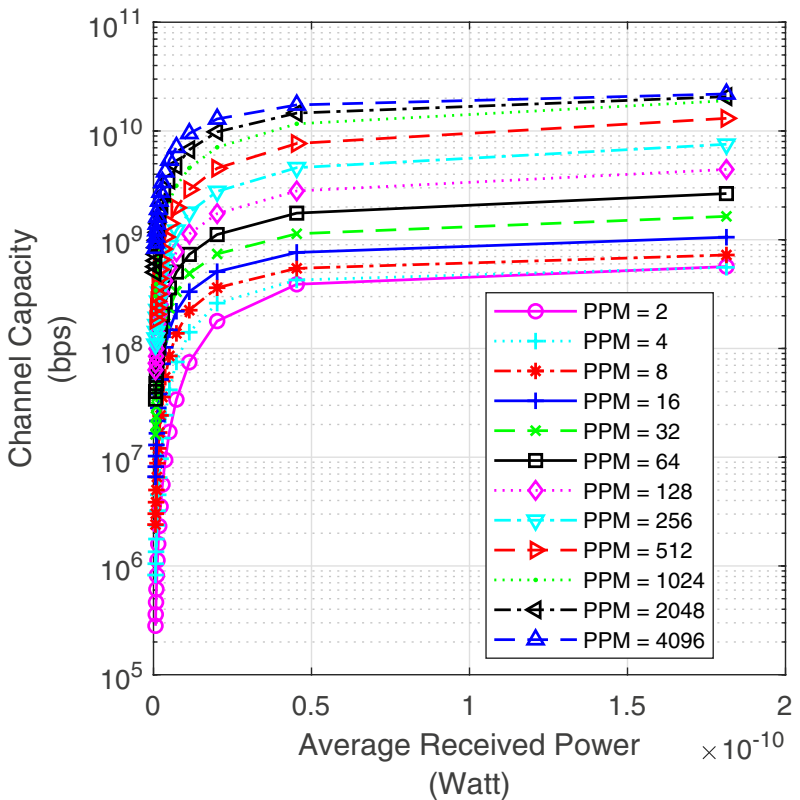

as shown in the charts for the data rate and data throughput for distance ranges less than 0.7AU, PPM orders $M<2048$ produce fairly better performance in throughput. This is 
Fig. 17 Capacity versus distance

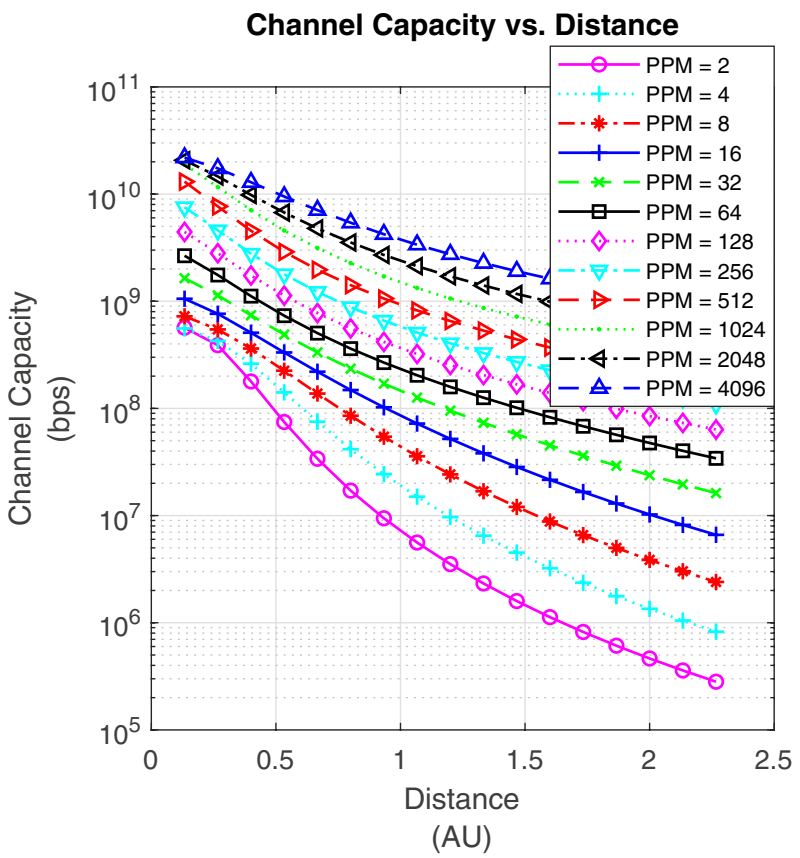

Fig. 18 Data rate versus distance

Data Rate vs. Distance

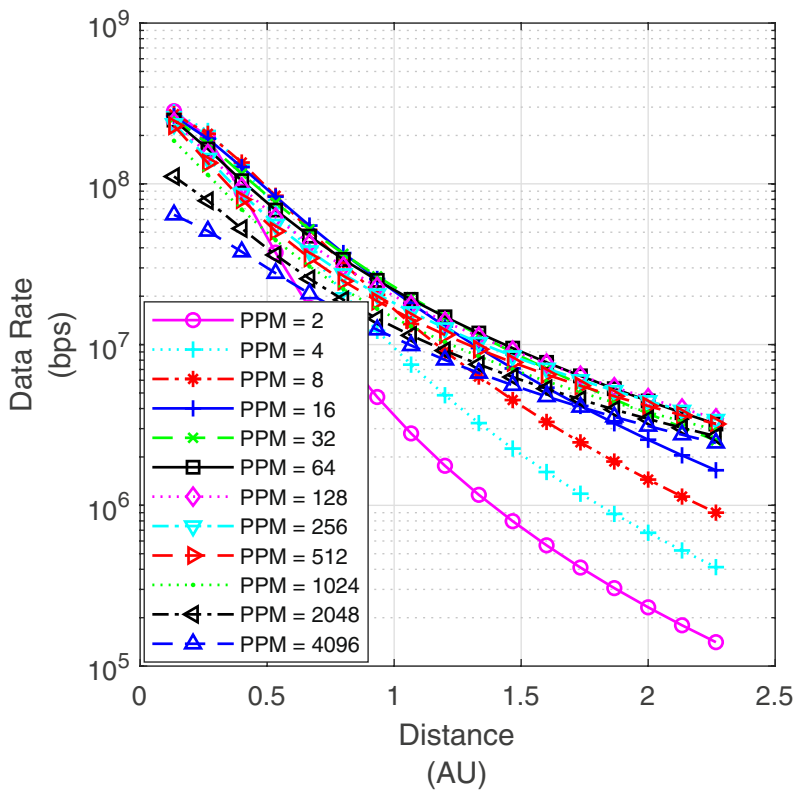

explained by the peak capacity constraint (saturation) that occurs due to an upper limit on the PPM order. Furthermore, for distance ranges more than 0.7AU, PPM orders from $M=2$ to $M=16$ produce degraded performances in data rate. Generally, it can be observed 
Fig. 19 Throughput versus distance
Data Throughput vs. Distance

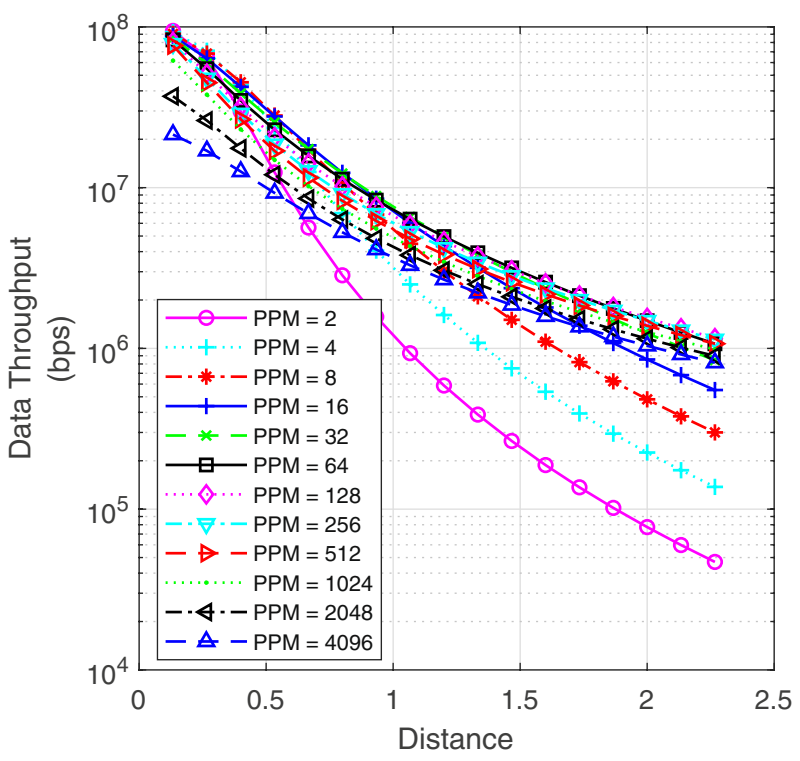

$(\mathrm{AU})$

that above $1 \mathrm{AU}$, the saturation limit on the data rate with respect to the upper limit constraint in PPM order (M) as explained earlier is depicted in Fig. 17 because increasing the PPM order from $M=32$ to $M=4096$ does not produce a sharp increment in the data rate compared to increasing the PPM order from $M=2$ to $M=32$. This also partly explains why very high PPM orders like $\mathrm{M}=2048$ or $\mathrm{M}=4096$ are not practically used in EarthMars missions. These very high PPM orders also come with other requirements such as very high computing processing resources and peak power. As stated in the introduction, an earth-mars mission lifetime is associated with varying distances over an orbital period ranging from $0.37 \mathrm{AU}$ (56 million $\mathrm{km}$ ) to $1.5 \mathrm{AU}$ (225 million $\mathrm{km}$ ); hence a robust PPM order chosen must take into account the performance of the data rate within this distance ranges and conditions. Consequently, all these deductions from Fig. 17 help to understand reasons why PPM orders of 64/128/256 are commonly used by many deep space missions among the range of $2-4096$ in order to achieve high data rates and optimum data rate. Although up to $M=256$ is used till now, due to different technical reasons (like compute processing resources and peak power), the possibility of using up to $M=1024$ in the future could show some further improvement, as can be seen in the simulation results.

\section{Results validation}

We would like to mention that up to now only lunar laser communication demonstration (LLCD) has been done by NASA and ESA as shown in literature (Cornwell 2014) for Moon-Earth missions. Consequently, the DSOC simulations and the investigation for Mars-Earth mission are even more important for the future. There is no real time experiment for a Mars-Earth mission; as far as research conducted in search of results for real time experiments in DSOC deployment during Mars-Earth missions. However, there is 
Fig. 20 Capacity versus distance

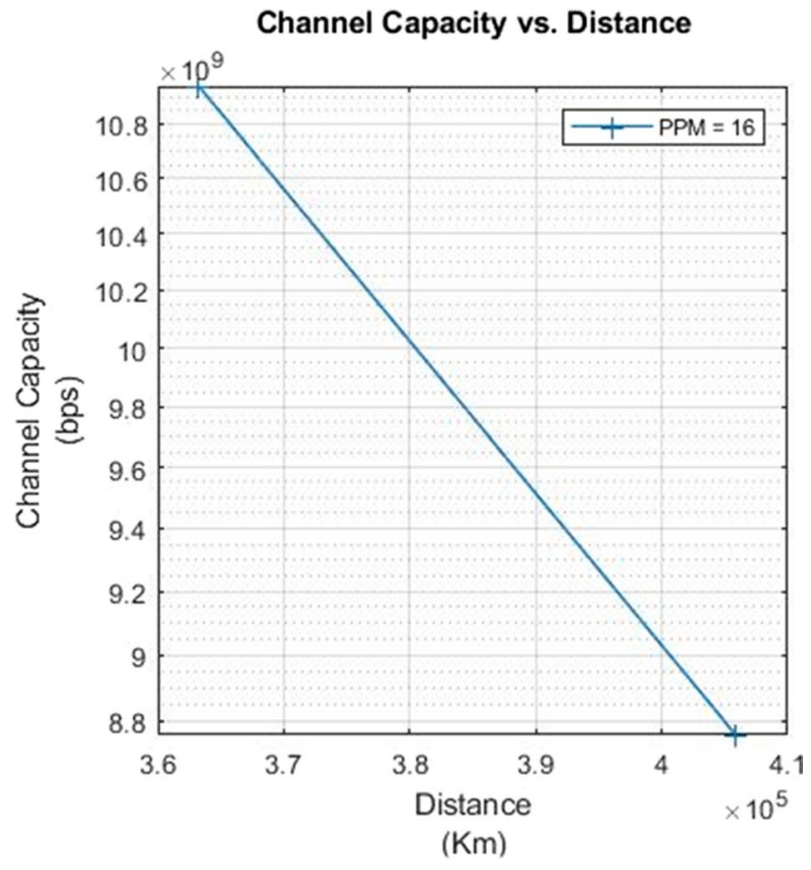

Fig. 21 Data rate versus distance

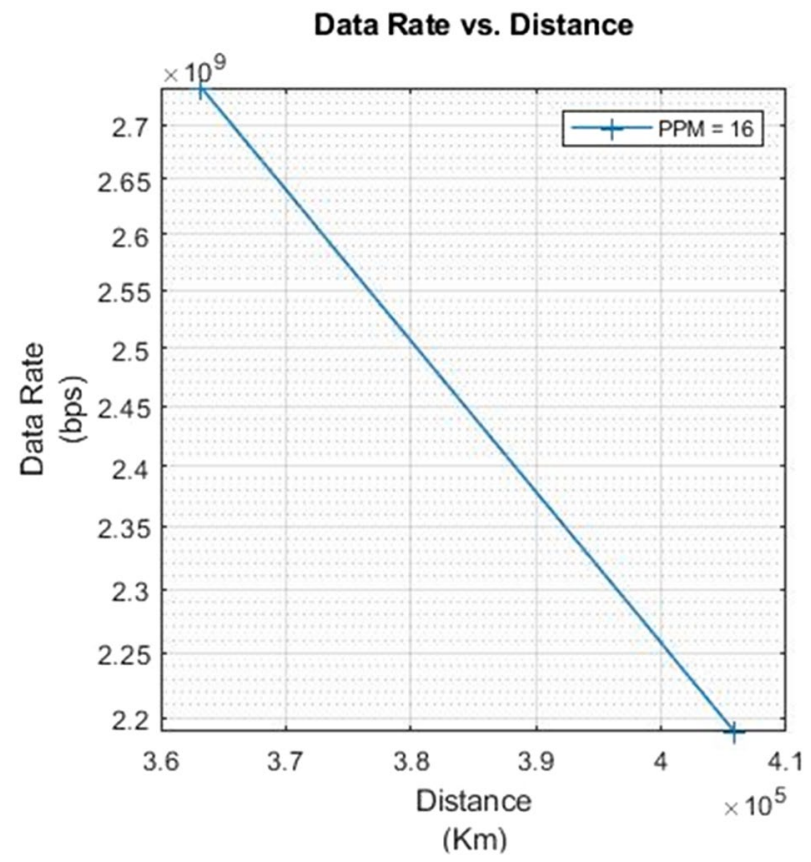

real time experiment in which laser communication was used for Moon-Earth missions by NASA and ESA as specified on page 22 (Cornwell 2014) of document issued by NASA titled "The Lunar Laser Communication Demonstration (LLCD)". Events gathered from 
Fig. 22 Throughput versus distance

Fig. 23 Photons/pulse versus distance
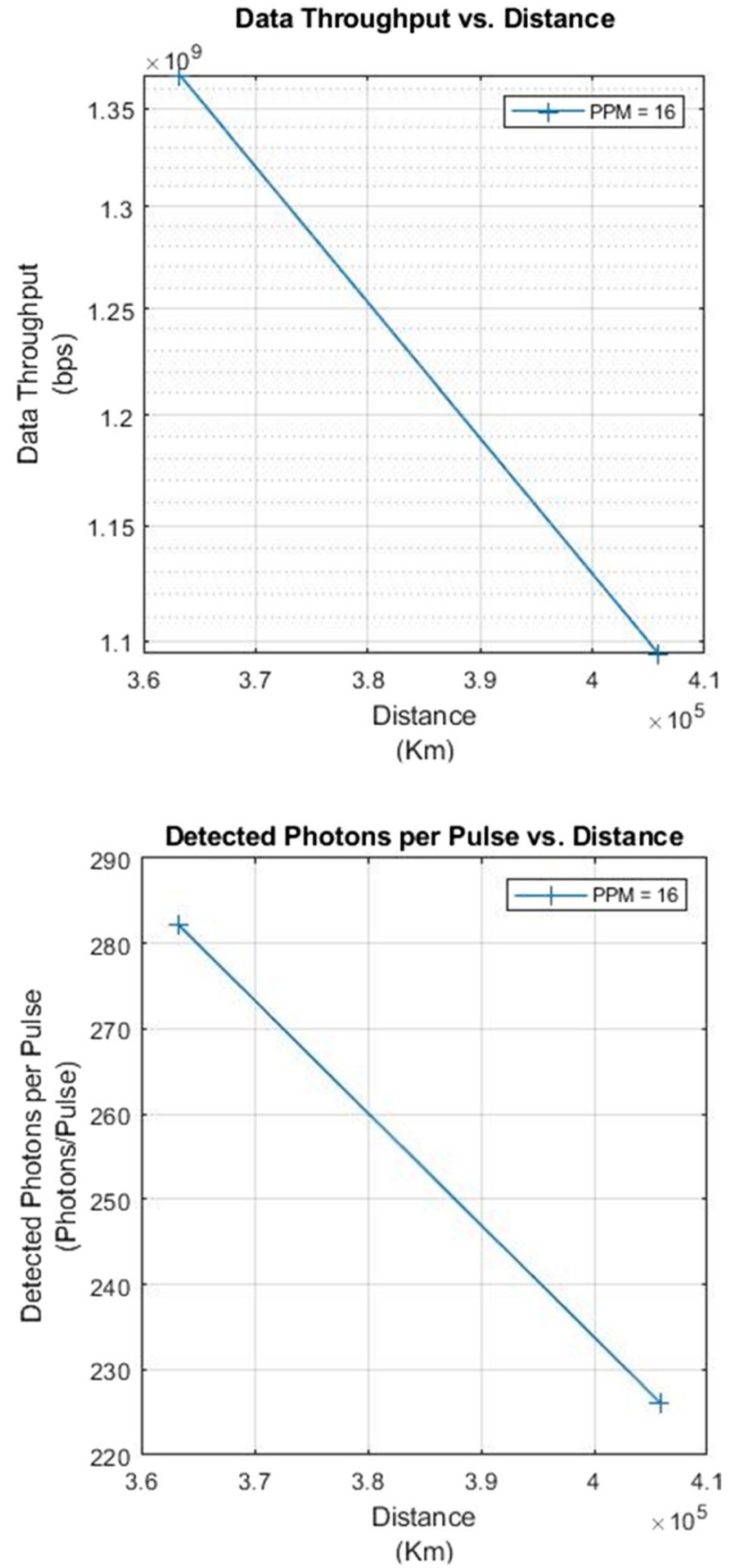

NASA and ESA projects towards the achievement of a real time experiment for DSOC Mars-Earth mission include: 
Fig. 24 BEP versus distance

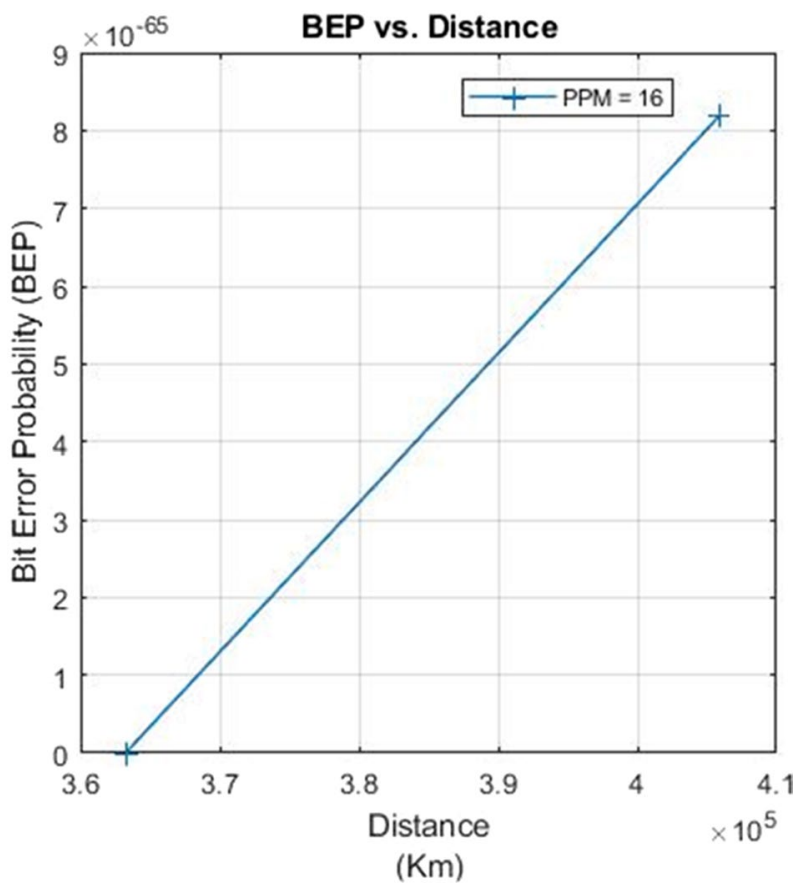

Fig. 25 BER versus distance

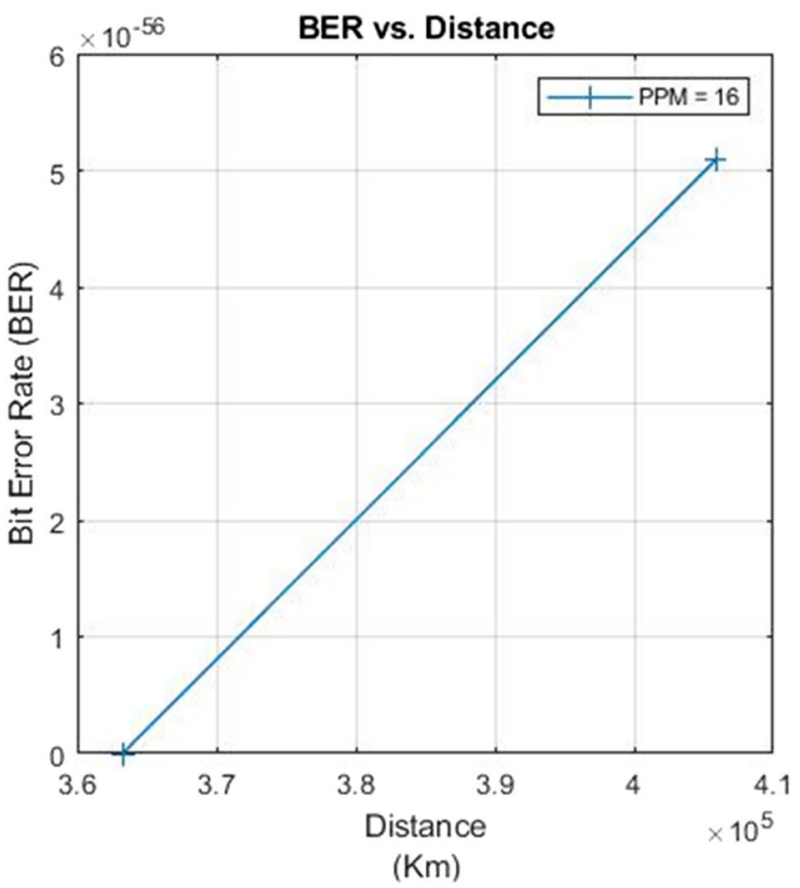


Fig. 26 SEP versus distance

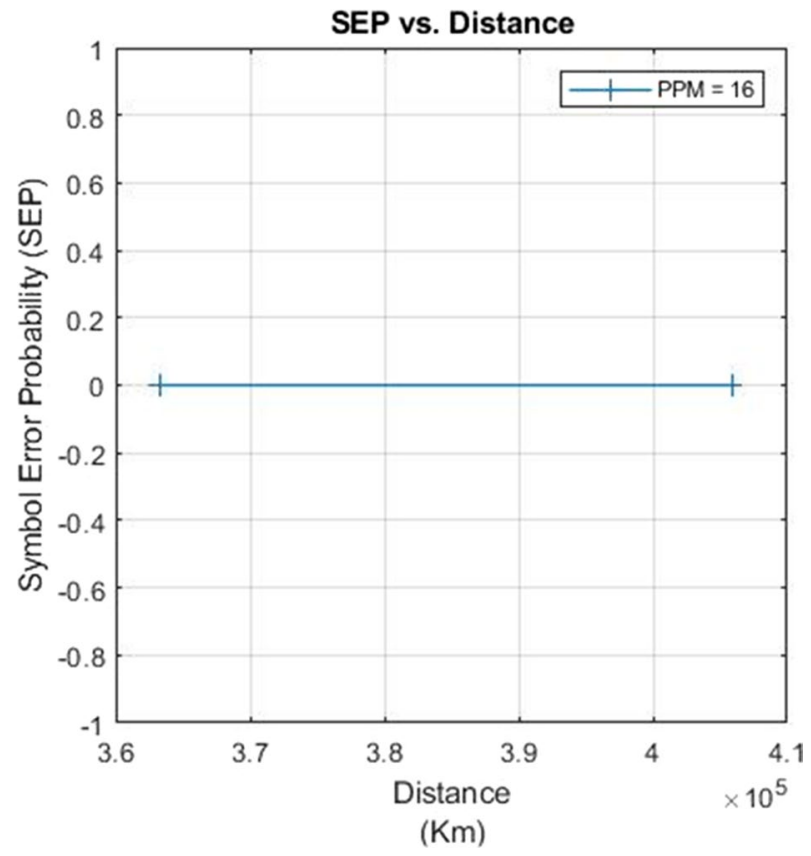

Fig. 27 SER versus distance

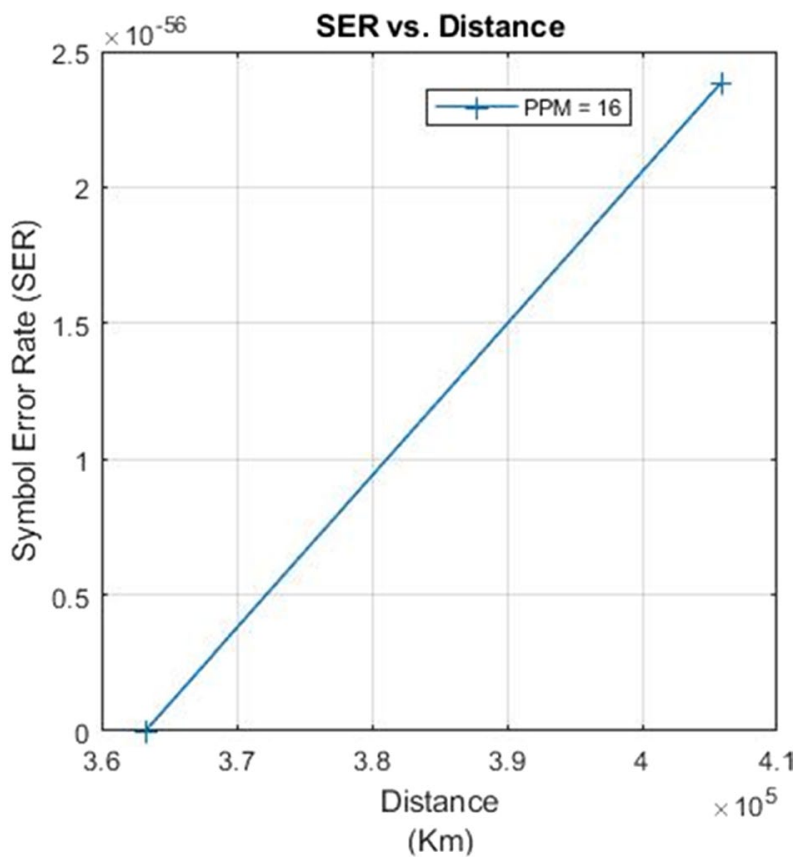

- One NASA planed project MTO (Mars Telecommunications Orbiter) with Laser Communications Demonstration beside X-band and Ka-Band RF transmission has been canceled; 
Table 2 Downlink throughput of real time experiment for LLCD (Cornwell 2014)

\begin{tabular}{lllc}
\hline $\begin{array}{l}\text { Description of test with } \\
\text { LLCD@622 Mbps }\end{array}$ & File Size (MB) & Duration (s) & $\begin{array}{l}\text { DL } \\
\text { Throughput } \\
\text { (Mbps) }\end{array}$ \\
\hline How high the Moon (mp3) & 2 & 0.013 & $1,230.77$ \\
Dark side of the Moon (CD) & 451.5 & 5.8 & 622.76 \\
Apollo 13 & 36,800 & 474 & 621.10 \\
U.S Library of Congress & $208,000,000$ & $2,674,800$ & 622.10 \\
\hline
\end{tabular}

- Another project is the NASA Psyche Project-in cooperation also with ESA-planned for 2022-2027;

Consequently, we have used a similar downlink DSOC simulation method to compare the results of NASA and ESA in their Moon-Earth downlink lunar laser communication with closest distance range $363,253 \mathrm{~km}$ and farthest distance $405,861 \mathrm{~km}$. Simulation was done for LLCD @ 622 Mbps using average transmit power $0.5 \mathrm{~W}$, 16-PPM and code rate of 0.5 as specified on page 5 (Cornwell 2014) of document issued by NASA titled "The Lunar Laser Communication Demonstration (LLCD)". Figures 20, 21, 22 and 23 respectively below show the channel capacity, data rate, throughput and detected photons/pulse plotted against distance during the downlink simulation of the Moon-Earth lunar communication link using a similar simulation method of the DSOC. Although Moon-Earth link is limited in distance range compared to a typical DSOC link, the downlink throughput from the simulation is $1100.00 \mathrm{Mbps}$ for the farthest Moon-Earth distance range and $1360.00 \mathrm{Mbps}$ for the closest MoonEarth distance range as shown in Fig. 22 below.

The BEP and BER in the LLCD are respectively shown in Figs. 24 and 25.

The SEP and SER in the LLCD are respectively shown in Figs. 26 and 27.

Table 2 is an extract from a presentation of NASA LLCD which shows the project accomplishment with real time experiment conducted for Moon-Earth mission by NASA and ESA. Downlink throughput achieved in real time experiment was from the range of 621.10 Mbps to as high as $1230.77 \mathrm{Mbps}$ depending on sky radiance variation and elevation. This is comparable to the average value of $1230.00 \mathrm{Mbps}$ determined from the downlink simulation of Moon-Earth link in Fig. 22 depending on sky radiance variation. This also validates the results from the downlink simulation done for the DSOC. The downlink data throughput achieved has high dependence on the PPM-order, code rate and existing sky radiance (background noise) at the receiver terminal.

Table 2 shows downlink throughput achievements from "The Lunar Laser Communication Demonstration (LLCD) @ 622 Mbps” issued by NASA [15, page 27].

\section{Conclusions}

Simulation results have confirmed that optimization to improve the received signal power (or received signal photons) can be accomplished by varying the PPM order. This can help to improve the ratio of the received signal photons to noise photons. Thus, increasing $\mathrm{M}$ can improve the capacity, bit error probability, bit error rate and achievable data rate of the DSOC system. Nevertheless, that leads to less bandwidth efficiency. In addition 
to the effective delivery of the signal to the detector, the performance of the optical link also depends on the receiver sensitivity (measured in terms of received photons per bit). Because of the high cost associated with increasing the transmit power and system aperture, improving the receiver sensitivity is an important factor in deep space optical communication system design. Thus optimizing the value of the PPM order (by choosing high M) could be the best way of achieving improved capacity, data rates and bit error probability. Nevertheless, the dead-time of the APD single photon counting receiver will limit the total slot width of the PPM codes, and should be considered in the design. Also the very high PPM order comes with requirements of high computing processing resources and also strict slot synchronization and error correction to ensure reliable data transmission and recovery.

Finally, simulation results have helped to explain reasons why PPM orders of 64/128/256 are commonly used by many deep space missions among the range of 2-4096 in order to achieve high data rates and optimum channel capacity. Even though up to $M=256$ is used till now, due to different technical reasons (like compute processing resources and peak power), the possibility of using up to $M=1024$ in the future could show some further improvement, as can be seen in the simulation results.

Supplementary Information The online version contains supplementary material available at https://doi. org/10.1007/s11082-021-03232-z.

Acknowledgements The author would like to thank Jon Hamkins, B. Moision, Michael K. Cheng and Robert Daddato for their friendly assistance by answering some questions in email correspondence at the initial stage of the writing of this paper which started as a team project under the supervision of Prof. Dr. ShunPing Chen. The authors appreciate many fruitful discussions with and helpful suggestions provided by Prof. Dr. Heinz Schmiedel.

Author contributions This manuscript simulates the impact of varying the pulse position modulation (PPM) order for a deep space optical communication system in order to optimize the data rates and bit error probabilities in varying operating points and conditions. It helps to understand reasons why PPM orders M of 64/128/256 are commonly used by many deep space missions among the range of 2-4096 in order to achieve high data rates at very low bit error probabilities. Even though up to $M=256$ is used till now, due to different technical reasons (like compute processing resources and peak power), the possibility of using up to $M=1024$ in the future could show some further improvement, as can be seen in the simulation results.

Funding Open Access funding enabled and organized by Projekt DEAL. Not Applicable.

Availability of data and material (data transparency) We declare that the data and material as well as simulation results in this manuscript were generated from our own work.

Code availability (software application or custom code) We declare that the code that was used to generate the simulations in this manuscript is the results of our own work and is available.

\section{Declarations}

Conflicts of interest The authors declare that they have no conflict of interest.

Open Access This article is licensed under a Creative Commons Attribution 4.0 International License, which permits use, sharing, adaptation, distribution and reproduction in any medium or format, as long as you give appropriate credit to the original author(s) and the source, provide a link to the Creative Commons licence, and indicate if changes were made. The images or other third party material in this article are included in the article's Creative Commons licence, unless indicated otherwise in a credit line to the material. If material is not included in the article's Creative Commons licence and your intended use is not permitted by statutory regulation or exceeds the permitted use, you will need to obtain permission directly from the copyright holder. To view a copy of this licence, visit http://creativecommons.org/licenses/by/4.0/. 


\section{References}

Arnon, S., Rotman, S.R., Kopeika, N.S.: Performance limitations of free-space optical communication satellite networks due to vibrations: direct detection digital mode. OE Opt. Eng. 36(11), 3148-3157 (1997)

Biswas, A., Piazzolla, S.: Deep-Space Optical Communications Downlink Budget from Mars: System Parameters, The Interplanetary Network Progress Report, pp. 1-38, June (2003)

Biswas, A., Wilson, K.E., Piazzolla, S., Wu, J., Farr, W.H.: Deep Space Optical Communications Link Availability and Data Volume, Deep Space Communications and Navigation Systems, Center of Excellence, Jet Propulsion Laboratory, California Institute of Technology, The International Society for Optical Engineering, pp. 1-10, June (2004)

Chen, C.-C.: Figure of Merit for Direct-Detection Optical Channels," The Telecommunications and Data Acquisition Progress Report 42-109, January-March 1992, Jet Propulsion Laboratory, Pasadena, California, pp. 136-151, May 15, (1992)

Cornwell, D.: The Lunar Laser Communication Demonstration (LLCD)", LLCD Mission Manager, NASA GSFC, Presentation to the JHU Aerospace Affinity Group, June 11, (2014)

Gallager, R.: Information Theory and Reliable Communication. Wiley, New York (1968)

Hamkins, J.: The Capacity of Avalanche Photodiode-Detected PulsePosition Modulation," The Telecommunications and Mission Operations Progress Report 42-138, April-June 1999, Jet Propulsion Laboratory, Pasadena, California, pp. 1-19, August 15, (1999)

Hamkins, J.: Accurate computation of the performance of M-ary orthogonal signaling on a discrete memoryless channel. IEEE Trans. Commun. 52(11), 1844-1845 (2004)

Helstrom, C.W.: Quantum Detection and Estimation Theory, Mathematics in Science and Engineering, vol. 123. Academic Press, New York (1976)

Hemmati, H.: Deep space optical communications, Deep Space Communications and Navigation Systems, Center of Excellence, Jet Propulsion Laboratory, California Institute of Technology, Publisher: Wiley (2006)

Hughes, L.W.: A simple upper bound on the error probability for orthogonal signals in white noise. IEEE Trans. Commun. 40, 670-671 (1992)

Moision, B., Hamkins, J.: Deep-Space Optical Communications Downlink Budget: Modulation and Coding," The Interplanetary Network Progress Report 42-154, April-June 2003, Jet Propulsion Laboratory, Pasadena, California, pp. 1-28, August 15 (2003)

Pierce, J.R., Posner, E.C., Rodemich, E.R.: The capacity of the photon counting channel. IEEE Trans. Inf. Theory. IT-27(1), 61-77 (1981)

Wyner, A.D.: "Capacity and error exponent for the direct detection photon channel-Part I. IEEE Trans. Inf. Theory 34(6), 1449-1961 (1988)

Publisher's Note Springer Nature remains neutral with regard to jurisdictional claims in published maps and institutional affiliations. 